Church History 78:2 (June 2009), 487-488.

(C) 2009, American Society of Church History

doi:10.1017/S0009640709000997 Printed in the USA

\title{
SOCIETY NOTICES
}

\begin{tabular}{|c|c|c|c|c|c|c|c|c|}
\hline ASCH BUDGET & $\begin{array}{l}02-03 \\
\text { Actual }\end{array}$ & $\begin{array}{l}03-04 \\
\text { Actual }\end{array}$ & $\begin{array}{l}04-05 \\
\text { Actual }\end{array}$ & $\begin{array}{l}05-06 \\
\text { Actual }\end{array}$ & $\begin{array}{l}06-07 \\
\text { Actual }\end{array}$ & $\begin{array}{l}07-08 \\
\text { Actual }\end{array}$ & $\begin{array}{c}08-09 \\
\text { YTD }\end{array}$ & $\begin{array}{c}08-09 \\
\text { Projected }\end{array}$ \\
\hline \multicolumn{9}{|l|}{ REVENUE } \\
\hline Member dues & 59,790 & 52,640 & 19,273 & 51,140 & 50,513 & 47,733 & 6,163 & 48,300 \\
\hline Conference Receipts & & 4,595 & 8,394 & 3,510 & 4,129 & 4,973 & 1,810 & 6,300 \\
\hline Subscriptions & 83,123 & 85,437 & 76,891 & 76,359 & 81,371 & 3,232 & 1,275 & 0 \\
\hline Advertising & 3,900 & 5,350 & 9,825 & 4,600 & 7,525 & 3,275 & 0 & 3,500 \\
\hline Sale of copies & 1,077 & 490 & 0 & 220 & 90 & 0 & 0 & 100 \\
\hline Member list & 250 & 1,075 & 250 & 250 & 0 & 850 & 500 & 1,000 \\
\hline Reprints & 200 & 250 & 50 & 150 & 0 & 50 & 150 & 100 \\
\hline Misc. receipts & 1,175 & 3,126 & 4,658 & 6,064 & 1,174 & 350 & 1,686 & 376 \\
\hline Interest & 7,289 & 300 & 0 & 0 & 0 & 0 & 0 & 0 \\
\hline Royalties & 9,362 & 5,471 & 8,111 & $18,030.19$ & 22,484 & $25,218.59$ & 697.4 & 28,000 \\
\hline Draw on investment & 2,407 & 2,900 & 47,287 & 2,050 & 15,662 & 20,455 & & 0 \\
\hline Subtotal & 168,573 & 161,635 & 174,739 & 162,373 & 182,948 & {$[106,227]$} & $12,281.27$ & 87,676 \\
\hline \multicolumn{9}{|l|}{ CUP Revenue } \\
\hline $\begin{array}{l}\text { Journal stipend (direct from } \\
\text { CUP) }\end{array}$ & & & & & & 20,000 & 0 & 20,000 \\
\hline Profit-sharing (CUP) & & & & & & 0 & 0 & 31,334 \\
\hline JSTOR Dissemination & & & & & & 6,000 & 0 & 6,000 \\
\hline \multirow[t]{3}{*}{ Total } & & & & & & 132,227 & $\mathbf{0}$ & 145,010 \\
\hline & $02-03$ & $03-04$ & $04-05$ & $05-06$ & $06-07$ & $07-08$ & $08-09$ & 08-09 \\
\hline & Actual & Actual & Actual & Actual & Actual & Actual & YTD & Projected \\
\hline \multicolumn{9}{|l|}{ CHURCH HISTORY-FSU } \\
\hline Editorial Staff & 35,569 & & & & & & & \\
\hline Coordinating Editors & & & 7,500 & 8,000 & 8,280 & 8,400 & 0 & 8,650 \\
\hline Sen. Grad Ass’t & & 3,294 & 10,522 & 9,938 & 10,237 & 9,939 & 4,141 & 15,200 \\
\hline Grad. Ass't & & 13,653 & & & & & & \\
\hline Book Review Ed. & & 3,649 & 4,124 & 4,310 & 4,310 & $4,309.68$ & 718 & 0 \\
\hline Copy Editor & & 14,286 & 15,788 & 14,981 & 13,666 & $16,149.90$ & 6,212 & 16,300 \\
\hline Office Assistant & & 193 & 0 & 0 & 0 & 200 & 0 & 0 \\
\hline Printing \& Distribution & 58,340 & & & & & & & \\
\hline Cadmus Press & & 50,295 & 49,328 & 55,785 & 59,467 & $16,405.33$ & 12,500 & \\
\hline Mailing & & 8,200 & 6,743 & 2,740 & 3,500 & 1,461 & 0 & 1,500 \\
\hline \multicolumn{9}{|l|}{ Travel \& Training } \\
\hline Training at Cadmus Press & & 797 & 0 & & 0 & 0 & 0 & 0 \\
\hline Grad. Ass't Training & & & 0 & 234 & 4,826 & 0 & 0 & 0 \\
\hline $\begin{array}{l}\text { Editors' Travel to } \\
\text { ASCH Mtg }\end{array}$ & & 1,758 & 3,031 & 2,380 & 4,686 & $2,549.41$ & 0 & 6,000 \\
\hline $\begin{array}{l}\text { Sen. Grad. Ass't-Ann. } \\
\text { Mtg. }\end{array}$ & & 815 & 835 & 334 & 428 & 850 & 0 & 2,500 \\
\hline $\begin{array}{l}\text { Copy Ed. Mtgs. With } \\
\text { CUP }\end{array}$ & & & & & & & & 2,300 \\
\hline \multicolumn{9}{|l|}{ Office } \\
\hline Supplies & & 1,442 & 1,116 & 1,089 & 96 & $3,446.70$ & 0 & 3,500 \\
\hline Postage & & 465 & 2,487 & 3,366 & 2,924 & $1,446.74$ & 0 & 1,500 \\
\hline Photocopying & & 64 & 0 & 0 & 0 & 0.00 & 0 & 0 \\
\hline \multicolumn{9}{|l|}{ Technology } \\
\hline Computer Depreciation & & & 500 & 500 & 500 & 500 & 250 & 500 \\
\hline Scanner Depreciation & & & 100 & 100 & 100 & 0 & 0 & 0 \\
\hline Printer Depreciation & & & 333 & 333 & 333 & 300 & 150 & 300 \\
\hline Repairs & & & 1,000 & 1,000 & 1,000 & 500 & 0 & 500 \\
\hline FSU maintenance fee & & & 500 & 500 & 500 & 1,500 & 0 & 1,500 \\
\hline Consultants/Hospitality & & & 1,000 & 1,000 & 1,000 & 800 & 0 & 800 \\
\hline Moving Costs & & 1,114 & 2,500 & 0 & 0 & 0 & & 0 \\
\hline Subtotal & $\mathbf{9 3 , 9 0 9}$ & 100,024 & 107,407 & 106,589 & 115,853 & 68,858 & 23,972 & 61,060 \\
\hline
\end{tabular}




\begin{tabular}{|c|c|c|c|c|c|c|c|c|}
\hline YDS/Baylor & $\begin{array}{l}02-03 \\
\text { Actual }\end{array}$ & $\begin{array}{l}03-04 \\
\text { Actual }\end{array}$ & $\begin{array}{l}04-05 \\
\text { Actual }\end{array}$ & $\begin{array}{l}05-06 \\
\text { Actual }\end{array}$ & $\begin{array}{l}06-07 \\
\text { Actual }\end{array}$ & $\begin{array}{l}07-08 \\
\text { Actual }\end{array}$ & $\begin{array}{c}08-09 \\
\text { YTD }\end{array}$ & $\begin{array}{c}08-09 \\
\text { Projected }\end{array}$ \\
\hline Executive Secretary & 19,500 & & & & & & & \\
\hline Salary & & 12,106 & 13,050 & $11,981.28$ & $11,981.28$ & $11,981.28$ & $4,992.20$ & 18,000 \\
\hline Travel-ASCH & & 2,074 & 2,445 & $1,739.99$ & 3,940 & 950.42 & & 2,500 \\
\hline Travel-ASCH-related & & 176 & 0 & 0 & 0 & 0 & & 500 \\
\hline Ass't to Exec. Secretary & 7,500 & & & & & & & \\
\hline Salary & & 3,722 & 5,382 & $5,421.51$ & 5,851 & $5,071.17$ & 1918.25 & 7,500 \\
\hline Travel & & 441 & 2,147 & 965.35 & 3,977 & 429.03 & & 2,500 \\
\hline Postage \& Supplies & 15,067 & 3,163 & 260 & 38 & 83 & 99 & 102 & 100 \\
\hline Accountant Fees & 2,300 & 2,520 & 975 & 4,050 & 2,788 & 2,000 & & 2,000 \\
\hline Payroll Taxes & 6,806 & & & & & & & \\
\hline Federal & & 6,173 & 8,194 & $8,087.72$ & 7,278 & 7,094 & 2,903 & 7,100 \\
\hline NJ State & & 1,008 & 164 & 10.17 & 0 & 0 & & 0 \\
\hline CT State & & 173 & 855 & 1273.53 & 897 & 868 & 505 & 900 \\
\hline $\begin{array}{l}\text { Computer Hardware/ } \\
\text { Software }\end{array}$ & 1,656 & 0 & 0 & 182 & 0 & 0 & & 0 \\
\hline Website & & 1,205 & 4,179 & $2,792.55$ & 1,492 & $1,274.40$ & 149.7 & 1,500 \\
\hline Telephone & 1,008 & 697 & 0 & 0 & 0 & 0 & 20 & 0 \\
\hline $\begin{array}{l}\text { Workmen's Comp. } \\
\text { Insurance }\end{array}$ & 475 & 8 & 0 & 0 & 0 & 0 & & 0 \\
\hline Penalty \& Interest & 0 & 0 & 0 & 156 & 0 & 0 & & 0 \\
\hline Depreciation & 0 & 0 & 0 & 0 & 0 & 0 & & 0 \\
\hline Bank Charges & 0 & 0 & & & & & & \\
\hline $\begin{array}{l}\text { People's Commercial } \\
\text { Acc't }\end{array}$ & & & 342 & 575 & 745 & 639 & 250 & 700 \\
\hline $\begin{array}{l}\text { Bank1 Online Merchant } \\
\text { Acc't }\end{array}$ & & & 636 & 1,281 & 817 & $1,300.55$ & 367 & 1,300 \\
\hline Advanta & & & & & & $1,156.25$ & 251.4 & 1,200 \\
\hline License \& Fees & 10 & 0 & 0 & 0 & 0 & 0 & & 0 \\
\hline Copyright & 150 & 30 & 135 & 0 & 270 & 0 & & 0 \\
\hline Refunds \& Cancellations & 0 & 291 & 180 & 90 & 90 & 0 & & 0 \\
\hline Subtotal & 54,472 & 39,961 & 38,944 & 38,645 & 40,209 & 32,864 & $11,458.78$ & 45,800 \\
\hline CONFERENCES/ & $02-03$ & $03-04$ & $04-05$ & $05-06$ & $06-07$ & $07-08$ & $08-09$ & $08-09$ \\
\hline OUTREACH & Actual & Actual & Actual & Actual & Actual & Actual & YTD & Projected \\
\hline Spring Conf. Fees & 7,387 & 5,250 & 4,916 & 0 & 2,927 & 498 & & 5,000 \\
\hline Winter Conf. Fees & 0 & 8,116 & 9,153 & 7,607 & 9,258 & $18,888.94$ & & 15,000 \\
\hline Advertising & 5,263 & 0 & & & & & & \\
\hline AHA & & 3,000 & 4,200 & 785 & 3,900 & 43 & 4,357 & 4,000 \\
\hline ACLS & & 990 & 990 & 0 & 1,088 & 1,238 & & 1,300 \\
\hline Prizes \& Awards & 3,000 & 1,523 & 2,250 & 1,550 & 3,500 & 3,000 & & 3,600 \\
\hline Student Travel Grants & & & 401 & 1,680 & 1,450 & 1,500 & & 2,500 \\
\hline Travel (Council) & 599 & 0 & 295 & 426 & 0 & 542 & 985 & 500 \\
\hline Lodging (Council) & 958 & 208 & 270 & 0 & 75 & 80 & & 250 \\
\hline Conference Programs & 0 & 2,584 & 5,913 & 5,092 & 4,688 & $4,714.97$ & 4,943 & 6,000 \\
\hline Subtotal & 17,207 & 21,670 & 28,388 & 17,139 & 26,886 & $30,504.96$ & 10,285 & 38,150 \\
\hline Total Expenses & $\$ 165,588$ & $\$ 161,635$ & 174,739 & 162,373 & 182,948 & $132,226.96$ & $45,715.78$ & 145,010 \\
\hline
\end{tabular}

